\title{
Detecting Subtle Vibrations Using Graphene-based Cellular Elastomers
}

M. Bulut Coskun ${ }^{a}, \dagger$, Ling Qiu $^{b}$, Md. Shamsul Arefin ${ }^{c}$, Adrian Neild ${ }^{a}$, Mehmet Yuce ${ }^{c}$, Dan $\mathrm{Li}^{b,}{ }^{*}$, Tuncay Alan ${ }^{a, *}$

a Laboratory for Micro Systems, Department of Mechanical and Aerospace Engineering, Monash University, Melbourne, VIC, 3800, Australia.

${ }^{\mathrm{b}}$ Department of Materials Science and Engineering, Monash University, VIC 3800, Australia.

${ }^{\mathrm{c}}$ Biomedical Integrated Circuits and Sensors Department of Electrical and Computer Engineering, Monash University, Melbourne, VIC, 3800, Australia.

KEYWORDS: graphene elastomer, flexible sensors, vibration sensor, accelerometers, microphones

\begin{abstract}
Ultralight graphene elastomer-based flexible sensors are developed to detect subtle vibrations within a broad frequency range. The same device can be employed as an accelerometer, tested within the experimental bandwidth of $20-300 \mathrm{~Hz}$ as well as a microphone, monitoring sound pressures from 300 to $20,000 \mathrm{~Hz}$. The sensing element does not contain any metal parts, making them undetectable by external sources and can provide a sensitivity of 2.6 $\mathrm{mV} / \mathrm{g}$, which is higher than those of rigid Si-based piezoresistive micro electro mechanical systems (MEMS).
\end{abstract}


Detection of subtle vibrations is important for a wide range of applications including rehabilitation ${ }^{1}$, activity recognition ${ }^{2}$, and biomechanical tracking ${ }^{3}$, all of which require compliant wearable sensory parts. Conventional micro electro mechanical systems (MEMS) consisting of accelerometers, gyroscopes and microphones were proposed to detect small changes in displacement, velocity and accelerations for body motion analysis ${ }^{4-5}$, fall detection ${ }^{6}$, to measure respiratory rates ${ }^{7}$ and perform electrocardiogram ${ }^{8}$, to name few. While these wellrefined devices can offer high sensitivity within a broad bandwidth, they require complex fabrication processes performed in sophisticated clean-room facilities and often complex circuitries. Additionally, traditional MEMS devices consist of rigid structures, which are unable to truly fulfil the requirements in emerging flexible electronics applications.

In recent years numerous flexible sensing elements using graphene, carbon nanotubes and metallic nanoparticles embedded in polymeric/elastomeric materials have been proposed as alternatives to MEMS based sensors. While such alternatives are suitable for quasi-static detection of classical mechanical quantities such as, strain ${ }^{9-12}$, force ${ }^{13}$ and pressure ${ }^{14-16}$, they have very limited response to detect vibrations, underperforming even in comparison to human skin (essentially a viscoelastic tactile sensor) which can comfortably detect dynamic excitations up to hundreds of Hertz ${ }^{17-18}$. The polymer elastomers employed in these sensors have relatively large elastic moduli and a poor response to high frequency excitations due to their viscoelasticity and poor mechanical recovery ${ }^{19-21}$. Although engineering of the architecture of the sensing components can potentially improve the detection sensitivity, the dynamic performance of previously reported polymer elastomer-based flexible sensors are still not comparable to those of conventional Si-based MEMS devices.

Recently, we ${ }^{22-23}$ and other groups ${ }^{24-29}$ have fabricated graphene-based elastomers (g. elastomers) by using graphene as a building block. This new class of graphene-based 
elastomeric material possesses a range unique features that are unattainable in conventional polymer elastomers. For example, graphene elastomers are extremely soft, their Young's modulus are 5,000 1,000,000 times lower than that of PDMS (a widely used polymer elastomer for flexible electronics); they are inherently piezoresistive and possess excellent response to dynamic force ${ }^{22-23}$. These unique features make graphene elastomer a promising candidate for sensing vibrations.

In this work, we investigate the vibration sensing capability of graphene elastomer, and demonstrate that the graphene elastomer can be used in sensing small vibrations with frequencies ranging from 20 to $20,000 \mathrm{~Hz}$, including uniaxial inertial loads $(20-300 \mathrm{~Hz})$ and sound waves within the audible frequency range (300 to 20,000 Hz). Their sensitivities are much higher than those of polymer elastomer-based sensors and even comparable to or better than the sophisticated Si-based piezoresistive MEMS devices ${ }^{30-40}$.

The devices were fabricated through previously developed freeze casting method ${ }^{23}$. (Details of the sample preparation and experimental procedures are provided in Supporting Information Section 1). The resultant cylinder shaped graphene elastomer (with $10 \mathrm{~mm}$ height and $12 \mathrm{~mm}$ diameter) revealed a honeycomb-like cellular structure and an ability to recover from large deformation (Figure $1(a-b))$. Interestingly, mechanical properties of the graphene elastomers can be tuned through the modification of density during the fabrication by simply altering the concentration of the graphene oxide dispersion. Our previous finding indicates that higher density graphene elastomer showed better dynamic response than lower density graphene elastomer ${ }^{22}$. (see Supporting Information Section 2 for the details.) Therefore, we focus on the sensing capabilities of a relatively dense graphene elastomer with $5 \mathrm{mg} / \mathrm{cm}^{3}$ density for the high frequency applications. Results for a low density graphene elastomerbased device have also been also provided for comparison (Figure S1). 

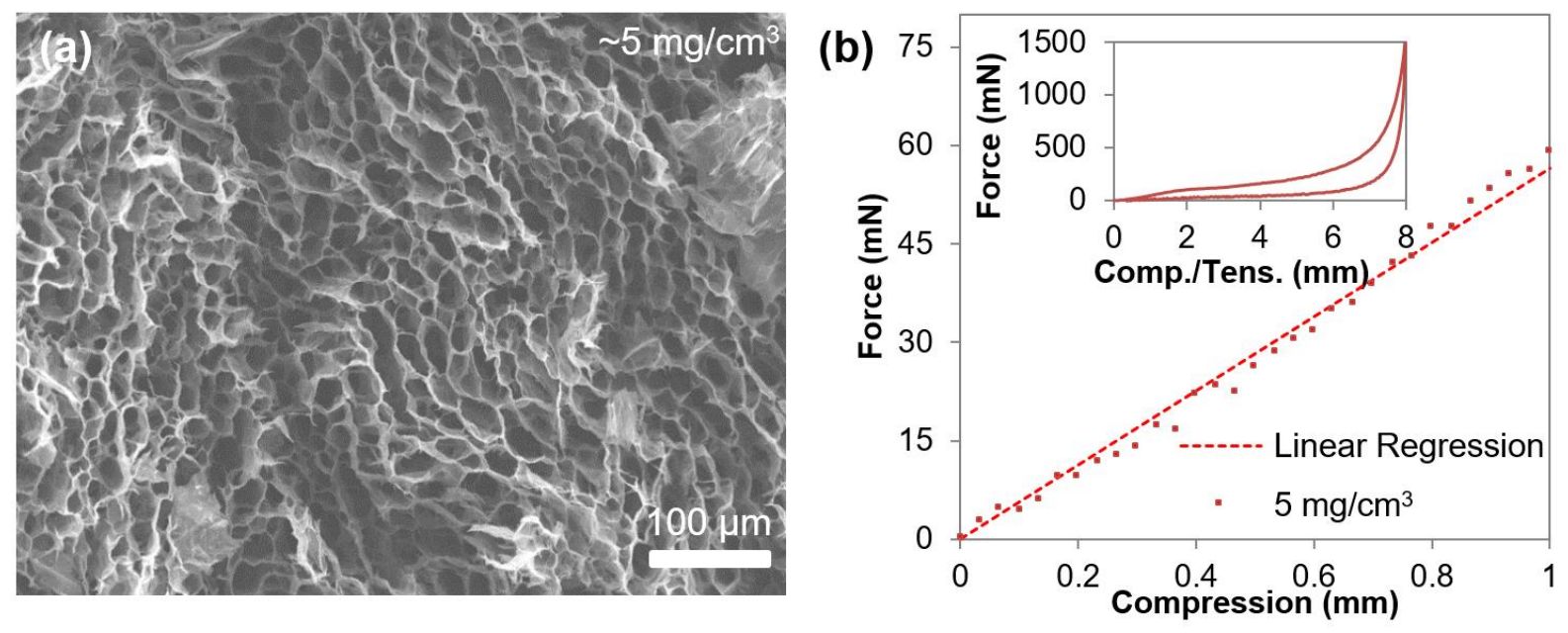

Figure 1 - (a) A Scanning Electron Microscope (SEM) Image showing the cellular structure of the graphene elastomer (g. elastomer) with a $5 \mathrm{mg} / \mathrm{cm}^{3}$ density. (b) Force-compression curve for $5 \mathrm{mg} / \mathrm{cm}^{3}$ device up to $10 \%$ compression. Inset shows the compression-release curve up to $80 \%$ where the device exhibits elastomeric behaviour.

To demonstrate the vibration sensing capability, a cylindrical proof mass was placed on top of the graphene elastomer-based sensor device to convert the vibration to the form of acceleration. As shown in Figure 2 (a), the device was excited with a sinusoidal loading generated by a commercial shaker with frequencies ranging from 20 to $300 \mathrm{~Hz}$ (limited by the reference commercial accelerometer's operation bandwidth). Figures 2 (b-d) show the response of the device under 40, 100 and $240 \mathrm{~Hz}$ actuation in the time domain. All three cases provided stable and consistent output and tracked the reference accelerometer well. As shown in Figure 2 (b), the phase difference between the device output and the commercial accelerometer is more pronounced at $40 \mathrm{kHz}$, which is within close proximity of the resonant frequency (see detailed discussion on the resonant frequency of the graphene elastomer in Supporting Information Section 4). 
(a)

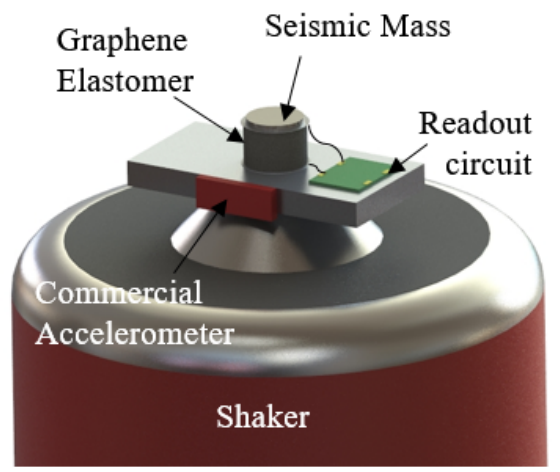

(c) $100 \mathrm{~Hz}$

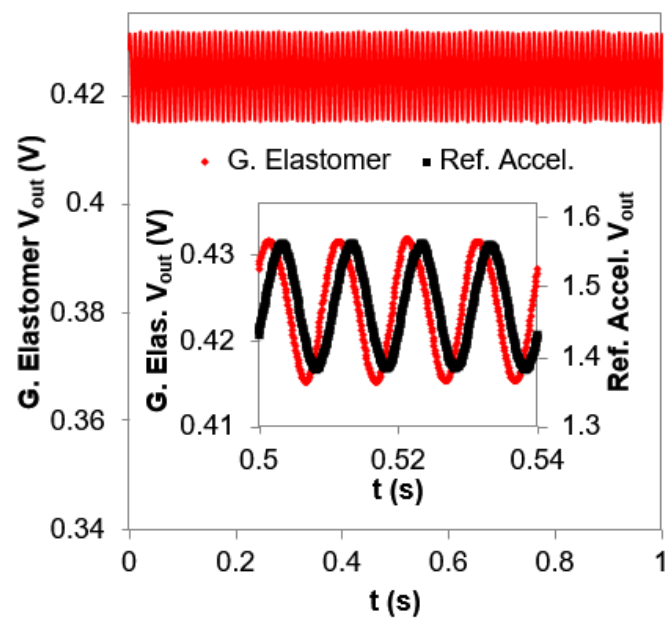

(b) $40 \mathrm{~Hz}$

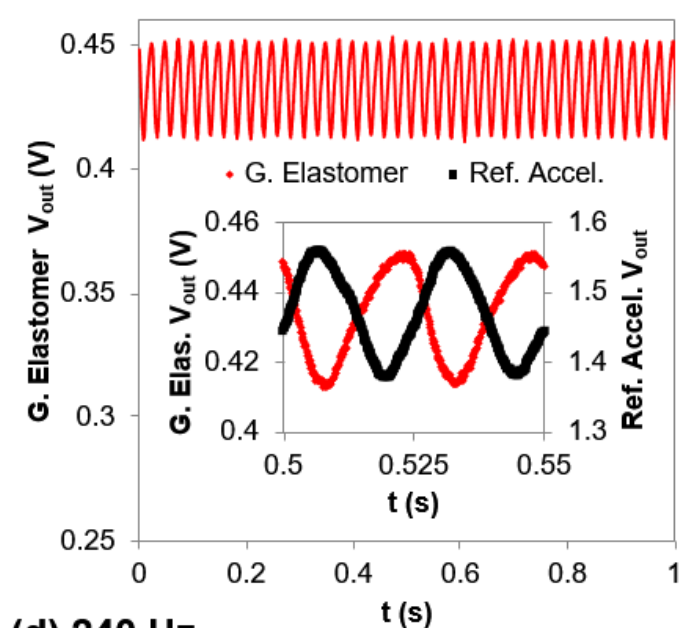

(d) $240 \mathrm{~Hz}$

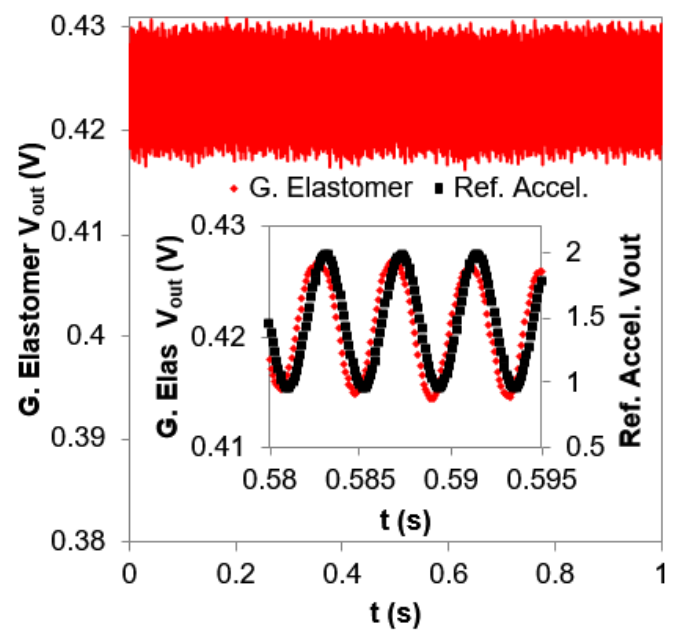

Figure 2-Schematic of the test setup for accelerometer experiments. (a) Voltage output of the graphene elastomer-based sensor (G. Elastomer) under sinusoidal excitation at (b) $40 \mathrm{~Hz}$, (c) $100 \mathrm{H}$ with $1 \mathrm{~g}_{\mathrm{pk}-\mathrm{pk}}$ and (d) $240 \mathrm{~Hz}$ with $6 \mathrm{~g}_{\mathrm{pk}-\mathrm{pk}}$ acceleration.

The graphene elastomer-based device can successfully measure up to $6 \mathrm{~g}$ peak-to-peak acceleration and exhibits a linear response to the changes in the amplitude of the acceleration when the acceleration is varied from $1 \mathrm{~g}_{\mathrm{pk}-\mathrm{pk}}$ to $6 \mathrm{~g}_{\mathrm{pk}-\mathrm{pk}}$ at several frequencies (Figure 3 (a)). Having known the peak-to-peak voltage output of the graphene elastomer for the corresponding acceleration, the acceleration sensitivity of the graphene elastomer is calculated for various excitation frequencies from the experiments where the device is excited by an acceleration of 
$1 \mathrm{~g}_{\mathrm{pk}-\mathrm{pk}}$ (Figure 3 (b)). Results show a high sensitivity at lower frequencies, which is due to low stiffness/proof mass ratio. This is also consistent with our theoretical approximation which indicates a resonance at $33 \mathrm{~Hz}$ considering the load-deformation curve in Figure 1 (b) (details available in the Supporting Information Section 4). The device sensitivity (S) reaches an average value of $2.6 \mathrm{mV} / \mathrm{g}$ above $150 \mathrm{~Hz}$ which is up to an order of magnitude higher without signal conditioning when compared to recent well-refined MEMS-based piezoresistive accelerometers ${ }^{30-40}$.

Apart from its excellent sensitivity, the graphene elastomer sensor also revealed excellent device performance, including good tracking comparability to the commercialized accelerometer and low noise level. The degree of linearity between the output signals of graphene elastomer device and the reference accelerometer was evaluated through a coherence analysis commonly used for accelerometer characterisation, and the result shows good correlation between graphene elastomer sensor and commercialized accelerometer ${ }^{34,41}$ (Figure S4). Additionally, to calculate the acceleration resolution of the device, its noise floor was analysed (Figure S5) to find the minimum detectable voltage bounded by the thermal noise. Utilizing the measured thermal noise floor for the device $(10.36 \mu \mathrm{V} / \sqrt{ }(\mathrm{Hz})$ and the corresponding device sensitivity (Figure 3 (b)), we have found that the graphene elastomerbased sensor devices can detect accelerations as small as $10 \mathrm{mg}$ (Figure S6). Importantly, all the data presented here was not filtered or amplified with the circuitry and raw output is presented in order to capture the intrinsic performance of the device. These results indicate excellent performance of the graphene elastomer-based accelerometer. 
(a)

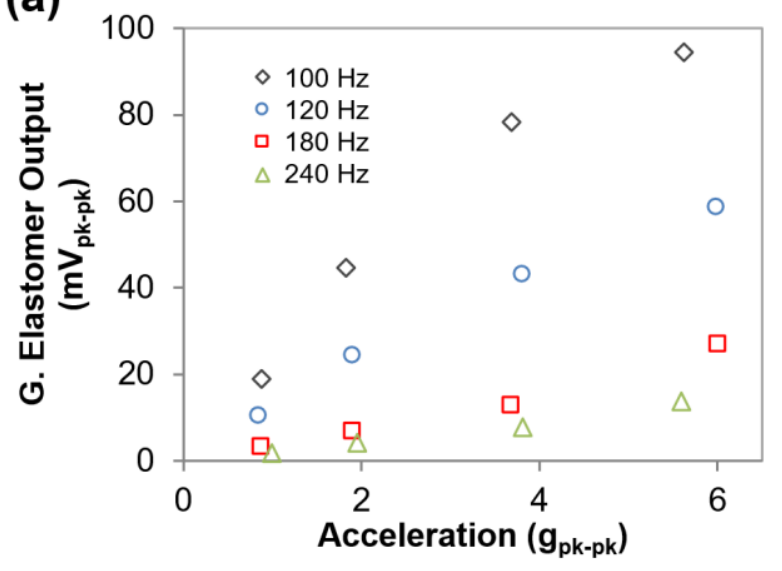

(b)

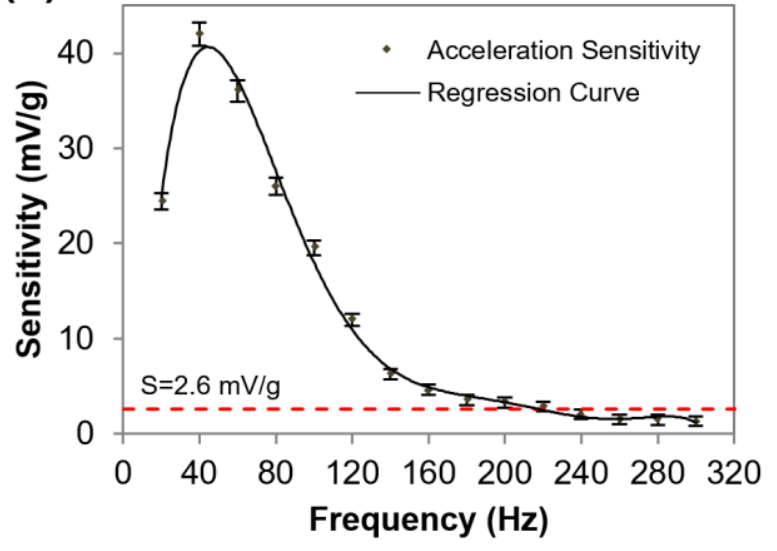

Figure 3 - (a) Voltage output (shown in logarithmic scale) of the graphene elastomer (G. Elastomer) $\left(5 \mathrm{mg} / \mathrm{cm}^{3}\right)$ at different acceleration amplitudes and frequencies with an absolute error of $\pm 0.527 \mathrm{mV}$. (b) Sensitivity of the device with respect to vibration frequency obtained from $1 g_{p k-p k}$ experiments. Absolute error originating from the measurement equipment is \pm 1.25 $\mathrm{mV} / \mathrm{g}$ at $40 \mathrm{~Hz}$ and less than $\pm 0.6 \mathrm{mV} / \mathrm{g}$ above $100 \mathrm{~Hz}$. Please see the Supporting Information Section 5 for the details regarding the measurement errors.

To further investigate the vibration sensing capability of the graphene elastomer at higher frequencies, the device was tested under sound pressures in the audible frequency range (up to $20 \mathrm{kHz}$ ) generated by a speaker positioned in the proximity, hence demonstrating its use as a microphone. For these experiments, we have again employed the same graphene elastomer-based device with identical density in the same configuration, but without the proof mass as the nature of the sound pressure is not inertial. The device can successfully track the reference microphone output when a constant amplitude sinusoidal sound wave is swept within the bandwidth of the speaker, 300-20,000 Hz (Figure 4 (b) and Supplementary Video 1). More interestingly, the device can successfully track sound waves applied simultaneously at different frequencies (Supplementary Video 2). Figure 4 (b) demonstrates the power spectrum of the graphene elastomer when excited with single tone sinusoidal sound waves with various frequencies resulting in a clear trend from 1 to $20 \mathrm{kHz}$ (the upper frequency limit defined by 
the bandwidth of the speaker used in the experiments). Moreover, we observe that graphene elastomer can successfully respond to the changes in the amplitude of sound waves, which have been varied at the same frequency (Figure 4 (c)). Although the sensor does not possess an optimized geometry for the microphone applications, it shows good dynamic response to sound waves, indicating promising performance in microphone application.

(a)

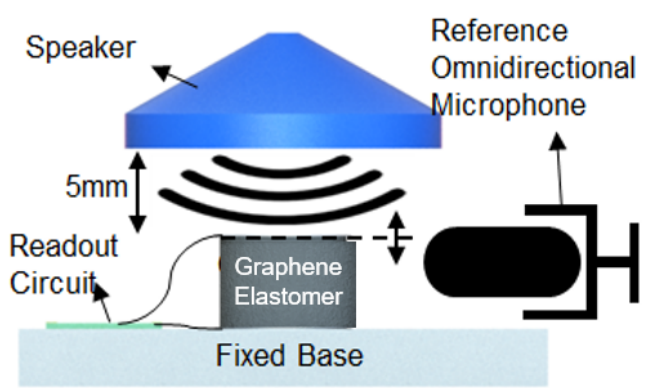

(b)

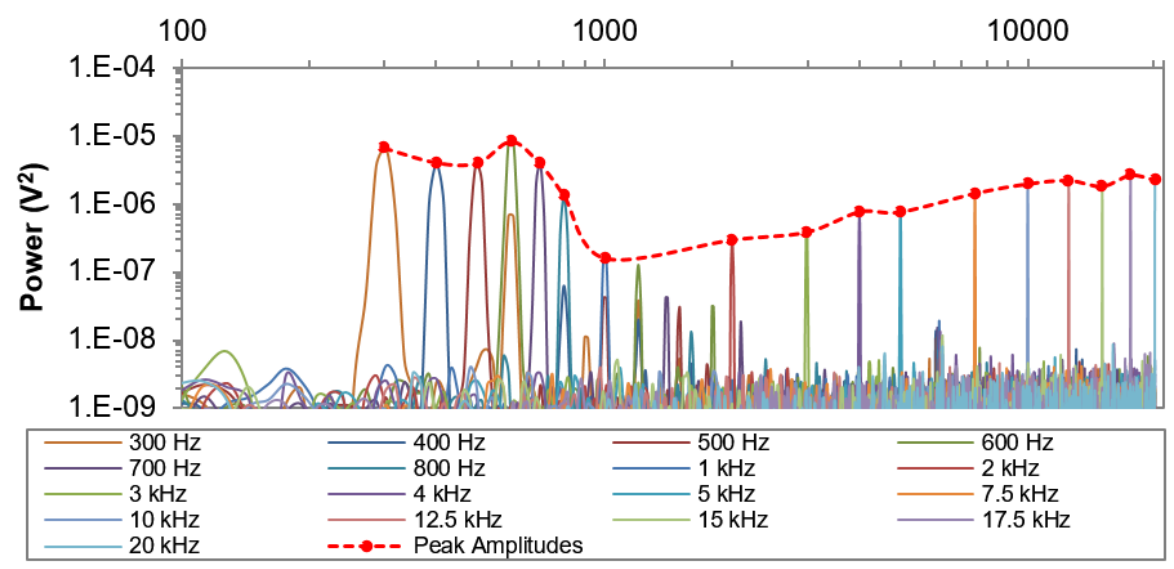

(c)

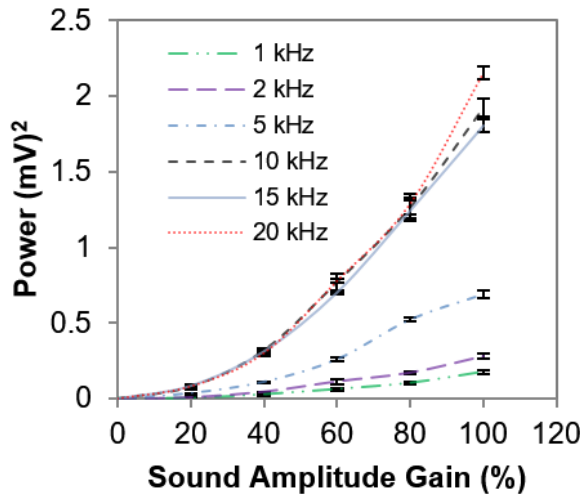

Frequency $(\mathrm{Hz})$

Figure 4-(a) A schematic of the test setup for microphone application. (b) Frequency response to sound pressures from $300 \mathrm{~Hz}$ to $20 \mathrm{kHz}$. The amplitude of the sound wave was kept constant and the gain of the speaker was set as $100 \%$ for this experiment. (c) Changes in the peak amplitudes of the power with respect to the variations in sound amplitude (varied by speaker gain) at various frequencies. Standard deviation based on 3 measurements have been presented in the form of error bars separately for each data points. Overall the relative error ranges from $\pm 2 \%$ to $\pm 8 \%$. 
The excellent vibration sensing capability of the graphene elastomer originates from its ultralow modulus and fast response nature. Owing to the ultralow modulus (ultralow stiffness) nature of the graphene elastomer, the subtle pressures induced by the inertial loading or the sound waves can result in sufficiently large deformations, leading to measureable changes in electrical resistance. Additionally, the stress transmission rate of graphene elastomer is comparable to those of dense polymer elastomers, enabling the fast response to high frequency dynamic vibration. Combining these unique features, graphene elastomer-based sensor can detect subtle vibration with a broad range frequency and provide outstanding performance in the application of accelerometer and microphone

In conclusion, we developed a soft vibration sensor based on the graphene elastomer, and further demonstrated its potential applications as an accelerometer and a microphone. The graphene elastomer-based accelerometer provides higher sensitivity and better synergy with wearable sensing applications than those of conventional rigid piezoresistive MEMS devices. Additionally, the graphene elastomer-based microphone is able to detect sound waves with frequency range of $300-20,000 \mathrm{~Hz}$. Noteworthy, the excellent vibration sensing capability is rarely found in flexible electronics made from the conventional polymer elastomers, as their viscoelastic behaviour and relatively high modulus limit their responsive behavior to subtle vibrations. This new class of soft vibration sensor is promising for novel flexible electronics applications, soft robotics and wearable sensing technologies. Finally, the devices contain practically no metallic parts, which prevents them from being discovered through external sources. This has a significant potential to be used in a number of systems requiring specialised undetectable sensing units (useful for instance in defence related applications). 


\section{ASSOCIATED CONTENT}

Supporting information for the following content has been included: experimental procedures; effect of density on the device sensitivity under dynamic loadings; cross-sample comparison: vibration response of two graphene elastomers with same density; theoretical estimation of the resonant frequency; standard deviation of the peak-to-peak voltage measurements and sensitivity; coherence analysis; thermal noise measurements; resolution of the accelerometer; readout circuit for the accelerometer application; readout circuit for the microphone application. Supporting Information (ESI) available free of charge via the Internet at http://pubs.acs.org."

\section{AUTHOR INFORMATION}

\section{Corresponding Authors}

*dan.li2@monash.edu

* tuncay.alan@monash.edu.au

\section{Present Addresses}

$\dagger$ Department of Mechanical Engineering, University of Texas at Dallas

\section{Author Contributions}

The manuscript was written through contributions of all authors. All authors have given approval to the final version of the manuscript.

\section{ACKNOWLEDGEMENTS}

Authors acknowledge support received from Monash University Postgraduate Publications Award. 
Abstract Figure

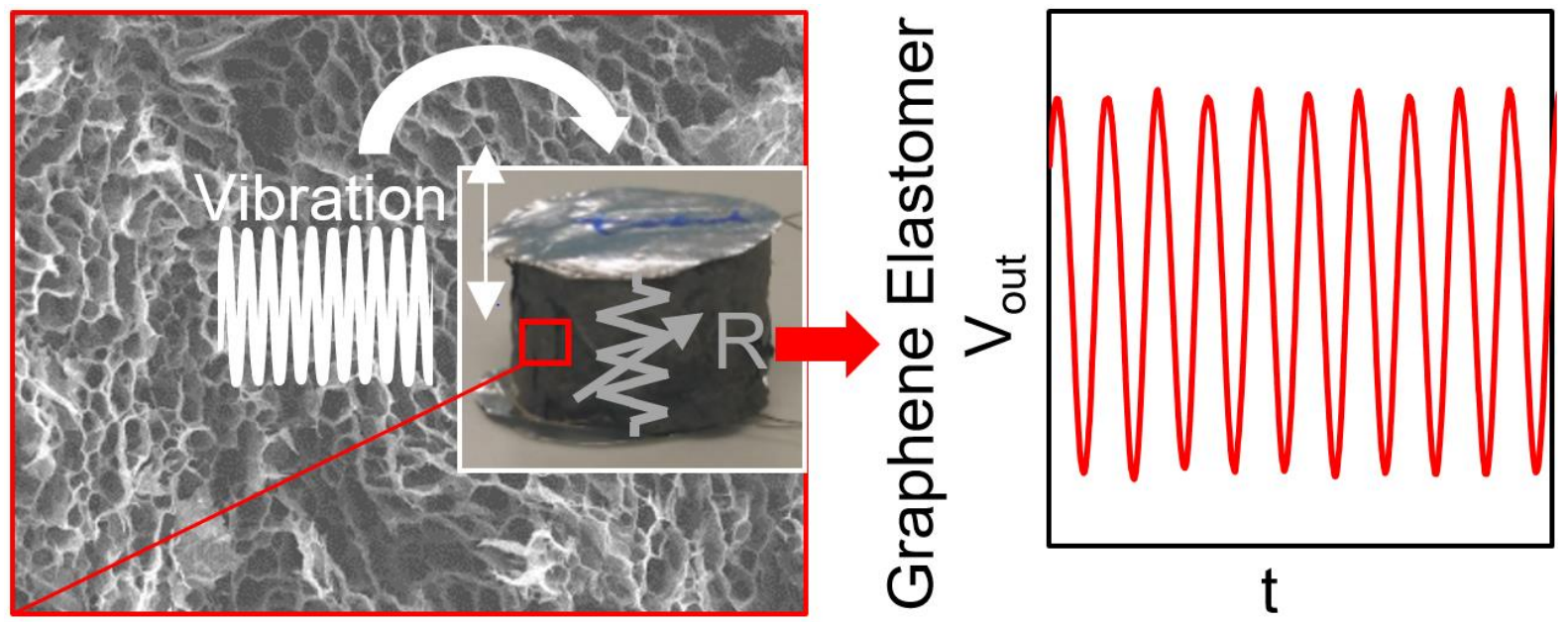




\section{REFERENCES}

1. Patel, S.; Park, H.; Bonato, P.; Chan, L.; Rodgers, M. A Review of Wearable Sensors and Systems with Application in Rehabilitation. J. Neuroeng. Rehabil. 2012, 9, 1.

2. Bao, L.; Intille, S. S. Activity Recognition from User-Annotated Acceleration Data. In Pervasive Computing, Springer: 2004, pp 1-17.

3. Scilingo, E. P.; Gemignani, A.; Paradiso, R.; Taccini, N.; Ghelarducci, B.; Rossi, D. D. Performance Evaluation of Sensing Fabrics for Monitoring Physiological and Biomechanical Variables. EEE Trans Inf Technol Biomed 2005, 9, 345-352.

4. D'Angelo, L. T.; Neuhaeuser, J.; Zhao, Y.; Lueth, T. C. Simple-Use-Sensor Set for Wearable Movement and Interaction Research. IEEE Sensors J. 2014, 14, 1207-1215.

5. Kan, Y.-C.; Chen, C.-K. A Wearable Inertial Sensor Node for Body Motion Analysis. IEEE Sensors J. 2012, 12, 651-657.

6. Shany, T.; Redmond, S. J.; Narayanan, M. R.; Lovell, N. H. Sensors-Based Wearable Systems for Monitoring of Human Movement and Falls. IEEE Sensors J. 2012, 12, 658-670.

7. Corbishley, P.; Rodríguez-Villegas, E. Breathing Detection: Towards a Miniaturized, Wearable, Battery-Operated Monitoring System. IEEE Trans. Biomed. Eng 2008, 55, 196-204.

8. Zhang, T.; Ser, W.; Daniel, G. Y. T.; Zhang, J.; Yu, J.; Chua, C.; Louis, I. In Sound Based Heart Rate Monitoring for Wearable Systems, 2010 International Conference on Body Sensor Networks, IEEE: 2010; pp 139-143.

9. Wang, Y.; Yang, R.; Shi, Z.; Zhang, L.; Shi, D.; Wang, E.; Zhang, G. Super-Elastic Graphene Ripples for Flexible Strain Sensors. ACS Nano 2011, 5, 3645-3650.

10. Li, X.; Zhang, R.; Yu, W.; Wang, K.; Wei, J.; Wu, D.; Cao, A.; Li, Z.; Cheng, Y.; Zheng, Q. Stretchable and Highly Sensitive Graphene-on-Polymer Strain Sensors. Sci. Rep. 2012, 2.

11. Hu, N.; Karube, Y.; Yan, C.; Masuda, Z.; Fukunaga, H. Tunneling Effect in a Polymer/Carbon Nanotube Nanocomposite Strain Sensor. Acta Mater. 2008, 56, 2929-2936.

12. Coskun, M. B.; Akbari, A.; Lai, D.; Neild, A.; Majumder, M.; Alan, T. Ultrasensitive Strain Sensor Produced by Direct Patterning of Liquid Crystals of Graphene Oxide on a Flexible Substrate. ACS Appl. Mater. Interfaces 2016, 8, 5 . 
13. Chun, S.; Kim, Y.; Jin, H.; Choi, E.; Lee, S.-B.; Park, W. A Graphene Force Sensor with PressureAmplifying Structure. Carbon 2014, 78, 601-608.

14. Tian, H.; Shu, Y.; Wang, X.-F.; Mohammad, M. A.; Bie, Z.; Xie, Q.-Y.; Li, C.; Mi, W.-T.; Yang, Y.; Ren, T.-L. A Graphene-Based Resistive Pressure Sensor with Record-High Sensitivity in a Wide Pressure Range. Sci. Rep. 2015, 5.

15. So, H.-M.; Sim, J. W.; Kwon, J.; Yun, J.; Baik, S.; Chang, W. S. Carbon Nanotube Based Pressure Sensor for Flexible Electronics. Materials Research Bulletin 2013, 48, 5036-5039.

16. Gong, S.; Schwalb, W.; Wang, Y.; Chen, Y.; Tang, Y.; Si, J.; Shirinzadeh, B.; Cheng, W. A Wearable and Highly Sensitive Pressure Sensor with Ultrathin Gold Nanowires. Nat. Commun. 2014, 5.

17. Johansson, R. S.; Landstro, U.; Lundstro, R. Responses of Mechanoreceptive Afferent Units in the Glabrous Skin of the Human Hand to Sinusoidal Skin Displacements. Brain Res. 1982, 244, 17-25.

18. Lundström, R. J. Responses of Mechanoreceptive Afferent Units in the Glabrous Skin of the Human Hand to Vibration. Scand. J. Work Environ. Health 1986, 413-416.

19. Hammock, M. L.; Chortos, A.; Tee, B. C. K.; Tok, J. B. H.; Bao, Z. 25th Anniversary Article: The Evolution of Electronic Skin (E - Skin): A Brief History, Design Considerations, and Recent Progress. Advanced Materials 2013, 25, 5997-6038.

20. Lakes, R. S. Viscoelastic Materials. Cambridge University Press: 2009.

21. Mark, J. E.; Erman, B.; Roland, M. The Science and Technology of Rubber. Academic press: 2013.

22. Qiu, L.; Bulut Coskun, M.; Tang, Y.; Liu, J. Z.; Alan, T.; Ding, J.; Truong, V. T.; Li, D. Ultrafast Dynamic Piezoresistive Response of Graphene - Based Cellular Elastomers. Adv. Mater. 2016, 28, $194-200$.

23. Qiu, L.; Liu, J. Z.; Chang, S. L.; Wu, Y.; Li, D. Biomimetic Superelastic Graphene-Based Cellular Monoliths. Nat. Commun. 2012, 3, 1241.

24. Barg, S.; Perez, F. M.; Ni, N.; do Vale Pereira, P.; Maher, R. C.; Garcia-Tuñon, E.; Eslava, S.; Agnoli, S.; Mattevi, C.; Saiz, E. Mesoscale Assembly of Chemically Modified Graphene into Complex Cellular Networks. Nat. Commun. 2014, 5.

25. Hu, H.; Zhao, Z.; Wan, W.; Gogotsi, Y.; Qiu, J. Ultralight and Highly Compressible Graphene Aerogels. Adv. Mater. 2013, 25, 2219-2223.

26. Li, Y.; Chen, J.; Huang, L.; Li, C.; Hong, J. D.; Shi, G. Highly Compressible Macroporous Graphene Monoliths Via an Improved Hydrothermal Process. Adv. Mater. 2014, 26, 4789-4793. 
27. Sun, H.; Xu, Z.; Gao, C. Multifunctional, Ultra - Flyweight, Synergistically Assembled Carbon Aerogels. Adv. Mater. 2013, 25, 2554-2560.

28. Wu, Y.; Yi, N.; Huang, L.; Zhang, T.; Fang, S.; Chang, H.; Li, N.; Oh, J.; Lee, J. A.; Kozlov, M. Three-Dimensionally Bonded Spongy Graphene Material with Super Compressive Elasticity and near-Zero Poisson's Ratio. Nat. Commun. 2015, 6.

29. Zhu, C.; Han, T. Y.-J.; Duoss, E. B.; Golobic, A. M.; Kuntz, J. D.; Spadaccini, C. M.; Worsley, M. A. Highly Compressible 3d Periodic Graphene Aerogel Microlattices. Nat. Commun. 2015, 6.

30. Li, Y.; Zheng, Q.; Hu, Y.; Xu, Y. Micromachined Piezoresistive Accelerometers Based on an Asymmetrically Gapped Cantilever. J. Microelectromech. Syst. 2011, 20, 83-94.

31. Kuells, R.; Nau, S.; Salk, M.; Thoma, K. Novel Piezoresistive High-G Accelerometer Geometry with Very High Sensitivity-Bandwidth Product. Sensors and Actuators A: Physical 2012, 182, 41-48.

32. Huang, S.; Li, X.; Song, Z.; Wang, Y.; Yang, H.; Che, L.; Jiao, J. A High-Performance Micromachined Piezoresistive Accelerometer with Axially Stressed Tiny Beams. J. Micromech. Microeng. 2005, 15, 993.

33. Hsieh, H.-S.; Chang, H.-C.; Hu, C.-F.; Cheng, C.-L.; Fang, W. A Novel Stress Isolation Guard-Ring Design for the Improvement of a Three-Axis Piezoresistive Accelerometer. J. Micromech. Microeng. 2011, 21, 105006.

34. Albarbar, A.; Badri, A.; Sinha, J. K.; Starr, A. Performance Evaluation of Mems Accelerometers. Measurement 2009, 42, 790-795.

35. Khir, M. H. M.; Qu, P.; Qu, H. A Low-Cost Cmos-Mems Piezoresistive Accelerometer with Large Proof Mass. Sensors 2011, 11, 7892-7907.

36. Roy, A. L.; Bhattacharyya, T. K. Design, Fabrication and Characterization of High Performance Soi Mems Piezoresistive Accelerometers. Microsystem Technologies 2015, 21, 55-63.

37. Roy, A. L.; Sarkar, H.; Dutta, A.; Bhattacharyya, T. K. A High Precision Soi Mems-Cmos $\pm 4 g$ Piezoresistive Accelerometer. Sensors and Actuators A: Physical 2014, 210, 77-85.

38. Sankar, A. R.; Das, S. A Very-Low Cross-Axis Sensitivity Piezoresistive Accelerometer with an Electroplated Gold Layer Atop a Thickness Reduced Proof Mass. Sensors and Actuators A: Physical 2013, 189, $125-133$.

39. Xu, Y.; Zhao, L.; Jiang, Z.; Ding, J.; Peng, N.; Zhao, Y. A Novel Piezoresistive Accelerometer with Spbs to Improve the Tradeoff between the Sensitivity and the Resonant Frequency. Sensors 2016, 16, 210. 
40. Zhang, L.; Lu, J.; Kurashima, Y.; Takagi, H.; Maeda, R. Case Studies of a Planar Piezoresistive Vibration Sensor: Measuring Transient Time History Signal Waves. Microelectron. Eng. 2016, 165, $27-31$.

41. Koka, A.; Sodano, H. A. High-Sensitivity Accelerometer Composed of Ultra-Long Vertically Aligned Barium Titanate Nanowire Arrays. Nat. Commun. 2013, 4. 